\title{
Estimation of the Disc Damage Likelihood Scale in primary open-angle glaucoma: the Glaucoma Stereo Analysis Study
}

\author{
Yasushi Kitaoka $^{1}$ - Masaki Tanito ${ }^{2,3} \cdot$ Yu Yokoyama $^{4} \cdot$ Koji Nitta $^{5} \cdot$ Maki Katai $^{6}$. \\ Kazuko Omodaka ${ }^{4}$. Toru Nakazawa ${ }^{4}$
}

Received: 19 August 2015 /Revised: 16 November 2015 / Accepted: 30 November 2015/Published online: 15 December 2015

(C) The Author(s) 2015. This article is published with open access at Springerlink.com

\begin{abstract}
Purpose The Glaucoma Stereo Analysis Study (GSAS), a cross-sectional multicenter collaborative study, used a stereo fundus camera (nonmyd WX) to assess various morphological parameters of the optic nerve head $(\mathrm{ONH})$ in glaucoma patients. We examined the associations between the Disc Damage Likelihood Scale (DDLS), a grading system for estimating glaucomatous $\mathrm{ONH}$ damage, and each parameter.

Methods The study included 187 eyes of 187 patients with primary open-angle glaucoma or normal-tension glaucoma. ONH morphological parameters including the DDLS stage were calculated with prototype analysis software. Three independent graders classified each optic disc appearance into four different types: focal ischemic, myopic glaucomatous, senile sclerotic, and generalized enlargement. The correlations between the DDLS and patient characteristics or each $\mathrm{ONH}$
\end{abstract}

Yasushi Kitaoka

kitaoka@marianna-u.ac.jp

1 Department of Ophthalmology, St. Marianna University School of Medicine, 2-16-1 Sugao Miyamae-ku,

Kawasaki, Kanagawa 216-8511, Japan

2 Division of Ophthalmology, Matsue Red Cross Hospital, 200 Horomachi, Matsue, Shimane 690-8506, Japan

3 Department of Ophthalmology, Shimane University Faculty of Medicine, Enya 89-1, Izumo, Shimane 693-8501, Japan

4 Department of Ophthalmology, Tohoku University Graduate School of Medicine, 1-1 Seiryo-machi, Aoba-ku, Sendai 980-8574, Japan

5 Department of Ophthalmology, Fukui-ken Saiseikai Hospital, 7-1 Funabashi, Wadanaka-machi, Fukui 918-8503, Japan

6 Department of Ophthalmology, Sapporo Teishin Hospital, 14-Jo 1-5-1 Kawazoe, Minami-ku, Sapporo 005-8798, Japan parameter were analyzed with Spearman's rank correlation coefficient.

Results The DDLS was correlated positively with baseline intraocular pressure and visual field pattern standard deviation, and negatively with visual field mean deviation. The DDLS was strongly correlated with vertical cup-to-disc ratio and horizontal cup-to-disc ratio positively, and with minimum rim-disc ratio negatively. The mean DDLS stage in the myopic glaucomatous type tended to be higher than the scores in other types.

Conclusion The DDLS obtained through three-dimensional ONH analysis correlates well with the severity of glaucomatous $\mathrm{ONH}$ and visual field damage.

Keywords Normal tension glaucoma · Primary open-angle glaucoma · Stereo fundus camera · Disc Damage Likelihood Scale $\cdot$ Optic nerve head

\section{Introduction}

Glaucomatous optic neuropathy (GON) is characterized by nerve fiber loss that can be recognized as thinning of the neuronal rim and enlargement of the excavation in the optic nerve head $(\mathrm{ONH})$. The cup-to-disc (C/D) ratio is a wellknown method to estimate the degree of excavation [1], but this ratio does not consider optic disc size. In a previous study using human donor eyes, the optic nerve fiber count increased significantly with enlarging optic disc size [2]. The Disc Damage Likelihood Scale (DDLS) is a method for estimating the degree of optic nerve damage which reflects disc size and has high intraobserver and interobserver reproducibility [3, 4]. The DDLS divides discs into three sizes, small $(<1.5 \mathrm{~mm})$, average $(1.5-2.0 \mathrm{~mm})$, and large $(>2.0 \mathrm{~mm})$, and is based on 
the width of the neuronal rim or the circumferential extent of absence of the neuronal rim $[3,4]$.

Although disc size is important for estimating GON damage, another pivotal factor for understanding GON damage is the ONH type. A previous study classified the glaucomatous ONH into four groups: focal ischemic (FI), myopic glaucomatous (MY), senile sclerotic (SS), and generalized enlargement (GE) [5]. Because the speed of progression of glaucomatous visual field defects may differ with each type $[6,7]$, identifying the type may be useful for predicting disease progression.

Topographic analysis with a simultaneous stereo fundus camera (nonmyd WX, Kowa Company, Ltd., Japan) is a noninvasive, noncontact imaging technique. The Glaucoma Stereo Analysis Study (GSAS) is a multicenter study using this technique to assess various morphological parameters of the ONH in Japanese glaucoma patients. The GSAS has recently demonstrated that significant negative associations were observed between the vertical C/D ratio and visual field mean deviation (MD) and between the disc tilt angle and refractive error [8]. In the present phase of the GSAS, we examined the relationships between the DDLS stage and patient characteristics or various ONH parameters and compared the mean DDLS stage among the four different disc types.

\section{Patients and methods}

The GSAS is a cross-sectional, multicenter, collaborative study, and we have recently reported the basic data including patient characteristics and representative $\mathrm{ONH}$ parameters [8]. It was approved by the institutional review boards of the Tohoku University Graduate School of Medicine, Shimane University Faculty of Medicine, Fukui-ken Saiseikai Hospital, Sapporo Teishin Hospital, and St. Marianna University School of Medicine. All experimental procedures were conducted in accordance with the tenets set forth in the Declaration of Helsinki. For this type of study, hospital-based and retrospective, formal consent is not required. All data collected from the participating institutions were analyzed anonymously.

One hundred and eighty-seven eyes of 187 patients with normal-tension glaucoma or primary open-angle glaucoma, comprising 100 men and 87 women aged (mean \pm standard deviation) $61 \pm 9$ years, were recruited into this study from five institutions: Tohoku University Hospital, the Hospital of Shimane University Faculty of Medicine, Fukui-ken Saiseikai Hospital, Sapporo Teishin Hospital, and the Hospital of St. Marianna University School of Medicine, as previously reported [8]. Briefly, the patients underwent full clinical ophthalmologic evaluation, including testing for refractive error and intraocular pressure (IOP) with Goldmann applanation tonometry, as well as slit lamp and fundus examinations. At least one measurement of pretreatment IOP (baseline IOP) was obtained retrospectively. Presurgical data on refractive error was also collected from eyes that had undergone refractive procedures such as cataract surgery. Visual field examinations with the Humphrey visual field analyzer (HFA; Carl Zeiss Meditec Inc., Dublin, CA, USA) were performed on all subjects within 6 months of recruitment. Data from at least six HFA examinations performed were also collected retrospectively for each patient. Additional inclusion criteria included: 1) best corrected visual acuity of 0.155 or better (LogMAR); 2) no congenital $\mathrm{ONH}$ anomalies; 3 ) $\mathrm{ONH}$ size within the typical normal range, defined as a disc-macula distance to disc diameter (DM/DD) ratio between approximately 2.4 and 3.0, 4 , no clinically apparent secondary cause of glaucoma, and no other disease affecting the visual field; 5) no history of intraocular surgery other than cataract or glaucoma surgery; 6) no history of cataract or glaucoma surgery in the previous three years; and 7) glaucomatous visual field loss more than $-12 \mathrm{~dB}$ MD. If both eyes met the inclusion criteria, the eye with more advanced glaucoma was selected [8].

The stereo pair of ONH photographs was obtained with a simultaneous stereo fundus camera (nonmyd WX). The nonmyd WX produces nonmydriatic fundus stereographs, and the built-in software (VK-2 WX, prototype version, Kowa Company, Ltd., Japan) automatically calculates ONH morphological parameters and the DDLS stage (nine stages: 0a, $0 \mathrm{~b}, 1,2,3,4,5,6,7)$ based on manually set contour lines for the $\mathrm{ONH}$ disc and cup, which in this study were determined by one of the authors (M.T.) while viewing the images stereoscopically. This determination was made according to the recommendations of the Japan Glaucoma Society Guideline for Glaucoma, 3rd edition, as previously reported [8].

Three independent assessors (T.N., K.O., and Y.Y.) classified each optic disc appearance into four different types: 1) FI discs showing localized tissue loss at the superior or inferior poles and a relatively intact neuroretinal rim elsewhere; 2) MY discs that had a tilted appearance and temporal crescent peripapillary atrophy (PPA) accompanied by additional evidence of glaucomatous damage, excluding discs with degenerative myopia; 3) SS discs with a saucerized shallow cup and diffuse neuroretinal rim tissue loss accompanied by surrounding PPA and choroidal sclerosis; and 4) GE discs characterized by a diffusely enlarged round cup and lack of localized defects of the neuroretinal rim with a previously reported Nicolela's clarification [5]. Discs that had features of multiple disc types were assigned to the most prominent one. If there was disagreement on the ONH classification among the three assessors, a consensual decision was adopted.

\section{Statistical analysis}

Statistical analysis was performed with JMP pro 10.02 (SAS Institute Inc., Cary, NC, USA) for Windows. Continuous 
variables were expressed as mean values \pm standard deviation. The Spearman rank correlation coefficient was used to determine correlations between patient characteristics and the $\mathrm{ONH}$ parameters obtained. In this analysis, ordinal data were treated as continuous. The level of significance was 0.05 in all statistical tests.

\section{Results}

A histogram of the distribution of the DDLS stage in the 187 patients is shown in Fig. 1. The average DDLS stage was $3.77 \pm 0.95$. The relationships between DDLS and patient characteristics are shown in Table 1. There was a weak, but significant positive correlation between the DDLS stage and baseline (pretreatment) IOP $(r=0.150$, $p=0.040$, Table 1) and a negative correlation between the DDLS stage and spherical equivalent refractive errors on both the test day $(r=-0.150, p=0.041)$ and pretreatment $(r=-0.183, p=0.012$, Table 1). Consistent with the findings in a previous study [3], a significant negative correlation was observed between the DDLS stage and MD $(r=-0.267, p<0.001$, Table 1) and a positive correlation was observed between the DDLS stage and visual field pattern standard deviation (PSD) $(r=0.233, p=0.001$, Table 1). The relationships between the DDLS and ONH parameters are shown in Table 2. Among the ONH morphological parameters, there were moderate positive correlations between the DDLS and vertical C/D ratio $(r=0.381, p<0.001$, Table 2$)$ and between the DDLS and horizontal C/D ratio $(r=0.292, p<0.001$, Table 2). In agreement with those findings, there was a strong negative correlation between the DDLS and minimum rim-to-disc (R/D) ratio $(r=-0.659, p<0.001$, Table 2). Similarly, there was a significant positive correlation between the DDLS and C/D area ratio $(r=0.292$, $p<0.001$, Table 2) and a moderate negative correlation between the DDLS and R/D area ratio $(r=-0.361$, $p<0.001$, Table 2).

Three independent assessors classified the 187 patients into four groups: FI $(n=34), \mathrm{GE}(n=38), \mathrm{MY}(n=96)$, and SS $(n=$ 19). The distribution of the DDLS stage in the four groups is shown in Table 3, and the mean DDLS stage in Table 4. The degrees of pretreatment spherical equivalent refractive errors in MY and SS were markedly less than those in FI and GE (Table 4). There was a significant difference in the mean DDLS stage between FI and MY (3.53 and 3.91, respectively, $p=0.0482$, Table 4). However, there was no significant difference in MD or baseline IOP among the four groups (Table 4). In addition, there was no significant difference in PSD between FI and MY, although it in GE was the least compared with those in the other groups (Table 4).

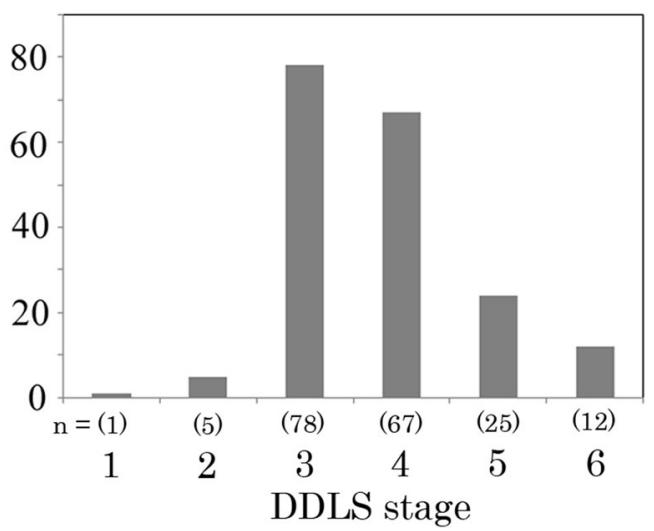

Fig. 1 Histogram of the distribution of the DDLS stage in patients in this study. DDLS=Disc Damage Likelihood Scale

\section{Discussion}

In optical coherence tomography (OCT) measurements, a fixed-diameter circular scan shows the difference in the distance between the scan point and the $\mathrm{ONH}$ margin dependent on the disc size, i.e., a smaller disc may have a long distance leading to a thinner retinal nerve fiber layer (RNFL) thickness, and a larger disc may display a thicker RNFL thickness. Therefore, one must be careful in interpreting the findings between disc size and RNFL thickness from OCT studies. Nonetheless, two different groups reported that RNFL

Table 1 Correlation between DDLS stage and glaucoma patient characteristics

Patient data

\begin{tabular}{|c|c|c|c|c|}
\hline \multirow[t]{2}{*}{ DDLS } & \multicolumn{4}{|c|}{ Age (years) } \\
\hline & $r$ & Lower $95 \%$ CI & Upper $95 \%$ CI & $p$ value \\
\hline & 0.032 & -0.112 & 0.175 & 0.666 \\
\hline & \multicolumn{4}{|c|}{ Corneal curvature radius on the test day (mm) } \\
\hline & -0.096 & -0.236 & 0.048 & 0.191 \\
\hline & \multicolumn{4}{|c|}{ Spherical refrative error on the test day (D) } \\
\hline & -0.156 & -0.293 & -0.013 & 0.033 \\
\hline & \multicolumn{4}{|c|}{ Pretreatment spherical refractive (D) } \\
\hline & -0.150 & -0.324 & -0.007 & 0.041 \\
\hline & \multicolumn{4}{|c|}{ Pretreatment spherical equivalent refractive error (D) } \\
\hline & -0.183 & -0.318 & -0.040 & 0.012 \\
\hline & \multicolumn{4}{|c|}{ Pretreatment IOP $(\mathrm{mmHg})$} \\
\hline & 0.150 & 0.007 & 0.288 & 0.040 \\
\hline & \multicolumn{4}{|c|}{$\mathrm{MD}(\mathrm{dB})$} \\
\hline & -0.267 & -0.395 & -0.128 & $<0.001$ \\
\hline & \multicolumn{4}{|c|}{$\operatorname{PSD}(\mathrm{dB})$} \\
\hline & 0.233 & 0.093 & 0.364 & 0.001 \\
\hline & \multicolumn{4}{|c|}{ Anti-glaucoma eye drops (Number) } \\
\hline & 0.150 & 0.007 & 0.287 & 0.040 \\
\hline
\end{tabular}

$D D L S$ Disc Damage Likelihood Scale, $M D$ mean deviation, $P S D$ pattern standard deviation 
Table 2 Correlation between DDLS stage and ONH parameters

Optic nerve head parameters

\begin{tabular}{|c|c|c|c|c|}
\hline \multirow[t]{2}{*}{ DDLS } & \multicolumn{4}{|c|}{ Vertical cup-disc ratio } \\
\hline & $r$ & Lower $95 \%$ CI & Upper $95 \%$ CI & $p$ value \\
\hline & 0.381 & 0.251 & 0.497 & $<0.001$ \\
\hline & \multicolumn{4}{|c|}{ Horizontal cup-disc ratio } \\
\hline & 0.292 & 0.155 & 0.418 & $<0.001$ \\
\hline & \multicolumn{4}{|c|}{ Minimum rim-disc ratio } \\
\hline & -0.659 & -0.733 & -0.570 & $<0.001$ \\
\hline & \multicolumn{4}{|c|}{ Superior minimum rim-disc ratio } \\
\hline & -0.242 & -0.373 & -0.102 & 0.001 \\
\hline & \multicolumn{4}{|c|}{ Angle of superior minimum rim-disc ratio } \\
\hline & -0.167 & -0.303 & -0.024 & 0.022 \\
\hline & \multicolumn{4}{|c|}{ Inferior minimum rim-disc ratio } \\
\hline & -0.528 & -0.625 & -0.417 & $<0.001$ \\
\hline & \multicolumn{4}{|c|}{ Superior rim width } \\
\hline & -0.240 & -0.371 & -0.100 & 0.001 \\
\hline & \multicolumn{4}{|c|}{ Inferior rim width } \\
\hline & -0.363 & -0.481 & -0.231 & $<0.001$ \\
\hline & \multicolumn{4}{|c|}{ Rim area } \\
\hline & -0.471 & -0.576 & -0.352 & $<0.001$ \\
\hline & \multicolumn{4}{|c|}{ Cup-disc area ratio } \\
\hline & 0.366 & 0.235 & 0.484 & $<0.001$ \\
\hline & \multicolumn{4}{|c|}{ Rim-disc area ratio } \\
\hline & -0.361 & -0.480 & -0.230 & $<0.001$ \\
\hline
\end{tabular}

$D D L S$ Disc Damage Likelihood Scale, $O N H$ optic nerve head

thickness significantly increased with an increase in optic disc size $[9,10]$. It was also reported that the Heidelberg Retina Tomograph (HRT) sensitivity required the definition of optic disc size classes or statistical correction for the size of the optic disc [11]. However, a recent study using the HRT II with the corrected effects of magnification on the disc measurements has shown that there was no significant association between RNFL thickness and optic disc area and suggested that the link between RNFL thickness and apparent disc size in the OCT study was probably due to magnification artifacts [12]. Although this does not support the assumption that this staging system reflects disc size, a human histological study that used a relatively high number of eyes ( 72 eyes from 56 donors) found increasing axon numbers with greater optic disc size [2], suggesting that the DDLS yields accurate estimates.

The DDLS is more reproducible than the C/D ratio system of estimating the amount of disc damage in patients with glaucoma $[3,4]$ and has been reported to be useful. For example, it was shown that the DDLS significantly correlated with all global and sectoral visual field indexes and with sectoral rim area HRT II measurements [13]. That study also showed that the DDLS stage correlated most strongly with superior and inferior regional data from HRT II and visual field measurements, and less well with temporal and nasal data [13]. The same group also demonstrated that the DDLS had the highest area under the curve (AUC) of 0.91 for predictive values of variables in differentiating glaucomatous from suspect or normal eyes rather than other factors including vertical $\mathrm{C} / \mathrm{D}$ ratio $(\mathrm{AUC}=0.81), \mathrm{MD}(\mathrm{AUC}=0.78), \mathrm{HPA}$ scoring $(\mathrm{AUC}=0.75)$, HRT II rim area $(\mathrm{AUC}=0.62)$, and Moorfield's regression analysis $(\mathrm{AUC}=0.54)$ [14]. In the present study, the DDLS stage was obtained automatically with the prototype analysis software. Using the same system, Han et al. have recently found good agreement (weighted kappa value, $0.59 \pm 0.03$ ) between DDLS stages obtained by stereo photography (nonmyd WX) and a glaucoma specialist [15]. There are some comparable findings between their study and our current study. For example, our present data show that the average DDLS stage was $3.77 \pm 0.95$ (all glaucoma patients, $n=187$ ), whereas it was $4.23 \pm 1.23$ in glaucoma patients in their study $(n=80)$ [15]. Because the average MD was $-4.71 \pm 3.26 \mathrm{~dB}$ and $-8.57 \pm 8.78 \mathrm{~dB}$ in our study and theirs, respectively, their study included more patients with severe glaucoma. It should be noted that our criteria for patient enrollment only included MD $>-12 \mathrm{~dB}$, as previously reported [8].

In the present study, there was a significant positive correlation between the DDLS stage and baseline IOP. This is consistent with the previous finding that progression is associated with the level of baseline IOP [16]. In addition, consistent with the results of previous studies [3, 17], we also found that the DDLS stage was significantly inversely correlated with the
Table 3 Distribution of DDLS stage in each disc type group

\begin{tabular}{llllll}
\hline DDLS & FI & GE & MY & SS & \\
\hline 1 & 0 & 0 & 1 & 0 & $1(0.5 \%)$ \\
2 & 0 & 1 & 2 & 2 & $5(2.7 \%)$ \\
3 & 19 & 16 & 37 & 6 & $78(41.7 \%)$ \\
4 & 12 & 16 & 32 & 7 & $67(35.8 \%)$ \\
5 & 3 & 4 & 13 & 4 & $24(12.8 \%)$ \\
6 & 0 & 1 & 11 & 0 & $12(6.4 \%)$ \\
& $34(18.2 \%)$ & $38(20.3 \%)$ & $96(51.3 \%)$ & $19(10.2 \%)$ & $187(100 \%)$ \\
\hline
\end{tabular}

$D D L S$ Disc Damage Likelihood Scale, $F I$ focal ischemic, $G E$ generalized enlargement, $M Y$ myopic glaucomatous, $S S$ senile sclerotic 
Table 4 Demographic data in each disc type group

\begin{tabular}{lllll}
\hline & FI & GE & MY & SS \\
\hline No. of patients & 34 & 38 & 96 & 19 \\
Baseline IOP (mmHg) & $16.1 \pm 4.9$ & $17.6 \pm 3.9$ & $16.8 \pm 3.5$ & $18.0 \pm 7.0$ \\
Pretreatment spherical equivalent & $-0.75 \pm 2.48$ & $-0.98 \pm 3.21$ & $-5.18 \pm 2.97$ & $-3.77 \pm 4.89$ \\
$\quad$ refractive error (D) & $p<0.0001$ & $p<0.0001$ vs. & $p<0.0001$ vs. FI & $p<0.005$ vs. FI \\
& vs. MY & MY & and GE & and GE \\
& $p<0.005$ vs. & $p<0.005$ vs. SS & & \\
Mean deviation (dB) & SS & & & \\
Pattern standard deviation $(\mathrm{dB})$ & $8.51 \pm 2.80$ & $-4.38 \pm 3.71$ & $-4.96 \pm 3.30$ & $-4.44 \pm 2.95$ \\
& $p<0.05$ vs. & $p<0.05$ vs. FI & $p<0.05$ vs. GE & \\
& GE & and MY & $3.99 \pm 3.63$ \\
Mean DDLS \pm SD & $3.53 \pm 0.66$ & $3.68 \pm 0.81$ & $3.91 \pm 1.08$ & $3.68 \pm 0.95$ \\
& $p<0.05$ vs. & & $p<0.05$ vs. FI & \\
& MY & & & \\
\hline
\end{tabular}

Data was expressed as mean values \pm standard deviation. Statistical significance was tested by ANOVA. FI focal ischemic, $G E$ generalized enlargement, $M Y$ myopic glaucomatous, $S S$ senile sclerotic, IOP intraocular pressure, DDLS Disc Damage Likelihood Scale
MD and positively correlated with the PSD, although the correlations were weak to moderate. These findings suggest that the DDLS stage reflects the degree of visual field damage that is measured and expressed by MD and PSD. Since the DDLS is based on the width of the neuronal rim or the circumferential extent of the absence of the neuronal rim, it is reasonable to find that the DDLS was correlated with both the vertical C/D and horizontal C/D ratios. This is in agreement with the findings from OCT data in a previous study [18]. That study also suggested that the DDLS was more useful than any other parameter acquired by OCT [18]. Furthermore, we demonstrated that the DDLS stage has a good inverse correlation with the minimum R/D ratio. Taking these results together, it is likely that the DDLS reflects the degree of ONH damage accurately.

Another noteworthy finding is the comparison of DDLS stages among the four different disc types. Myopia is a known risk factor for glaucoma in Asian [19, 20] and Latino [21] populations. A systematic review and meta-analysis demonstrated that individuals with myopia have an increased risk of developing OAG [22]. We found a negative correlation between the DDLS stage and spherical equivalent refractive errors. Therefore, one possibility is that myopia leads to a high degree of $\mathrm{ONH}$ damage. Among the glaucomatous $\mathrm{ONH}$ types classified using Nicolela's system, we found a significant difference in the mean DDLS stage between the FI and MY groups. MY patients had displayed significantly higher mean DDLS stage than FI patients. Another possibility is the overestimation of the DDLS in MY due to the slope in the temporal side. We found no significant differences in the mean MD and PSD between the FI and MY groups, although the MY group tended to have a higher mean MD value (MY, $-4.96 \pm 3.30 \mathrm{~dB}$; FI, -4.51 $\pm 2.80 \mathrm{~dB}$ ). To our knowledge, this is first study demonstrating the relationship between the DDLS stage and different glaucomatous ONH types.

One limitation of topographic analysis with a simultaneous stereo fundus camera is that it is not entirely automated software, and there might still be some bias caused by arbitrarily set margins in discs with atypical shapes, such as myopic discs with temporal crescent PPA or saucerized discs with shallow cupping, where it is difficult to define the margins. Nonetheless, determining the margins is more accurate in stereoscopic images than in monoscopic images.

In conclusion, the DDLS stage obtained through stereoscopic analysis was well correlated with $\mathrm{ONH}$ parameters and visual field damage. The DDLS tends to be categorized into higher stages in eyes with myopic disc appearance.

Acknowledgments The authors thank Mr. Toshiaki Nakagawa for several helpful comments.

\section{Compliance with ethical standards}

Funding No funding was received for this research.

Conflict of interest Authors Y.K., M.T., Y.Y., K.N., and T.N. received a speaker honorarium from Company Kowa. Authors M.T. and T.N. received financial support for the consultancies from Kowa. The Kowa had no role in the design or conduct of this research. Authors M.K. and K.O. certify that they have no affiliations with or involvement in any organization or entity with any financial interest (such as honoraria; educational grants; participation in speakers' bureaus; membership, employment, consultancies, stock ownership, or other equity interest; and expert testimony or patent-licensing arrangements), or non-financial interest (such as personal or professional relationships, affiliations, knowledge, or beliefs) in the subject matter or materials discussed in this manuscript. 
Ethical approval All procedures performed in studies involving human participants were in accordance with the ethical standards of the institutional and/or national research committee and with the $1964 \mathrm{Hel}-$ sinki declaration and its later amendments or comparable ethical standards.

Informed consent For this type of study, hospital-based and retrospective, formal consent is not required.

Open Access This article is distributed under the terms of the Creative Commons Attribution 4.0 International License (http:// creativecommons.org/licenses/by/4.0/), which permits unrestricted use, distribution, and reproduction in any medium, provided you give appropriate credit to the original author(s) and the source, provide a link to the Creative Commons license, and indicate if changes were made.

\section{References}

1. Armaly M (1967) Genetic determination of cup/disk ratio of the optic nerve. Arch Ophthalmol 78:35-43

2. Jonas JB, Schmidt AM, Müller-Bergh JA, Schlötzer-Schrehardt UM, Naumann GO (1992) Human optic nerve fiber count and optic disc size. Invest Ophthalmol Vis Sci 33:2012-2018

3. Bayer A, Harasymowycz P, Henderer JD, Steinmann WG, Spaeth GL (2002) Validity of a new disk grading scale for estimating glaucomatous damage: correlation with visual field damage. Am J Ophthalmol 133:758-763

4. Spaeth GL, Henderer J, Liu C, Kesen M, Altangerel U, Bayer A, Katz LJ, Myers J, Rhee D, Steinmann W (2002) The disc damage likelihood scale: reproducibility of a new method of estimating the amount of optic nerve damage caused by glaucoma. Trans Am Ophthalmol Soc 100:181-185

5. Nicolela MT, Drance SM (1996) Various glaucomatous optic nerve appearances: clinical correlations. Ophthalmology 103: 640-649

6. Nicolela MT, McCormick TA, Drance SM, Ferrier SN, LeBlanc RP, Chauhan BC (2003) Visual field and optic disc progression in patients with different types of optic disc damage: a longitudinal prospective study. Ophthalmology 110:2178-2184

7. Nakazawa T, Shimura M, Ryu M, Himori N, Nitta F, Omodaka K, Doi H, Yasui T, Fuse N, Nishida K (2012) Progression of visual field defects in eyes with different optic disc appearances in patients with normal tension glaucoma. J Glaucoma 21:426-430

8. Yokoyama Y, Tanito M, Nitta K, Katai M, Kitaoka Y, Omodaka K, Tsuda S, Nakagawa T, Nakazawa T (2014) Stereoscopic analysis of optic nerve head parameters in primary open angle glaucoma: the Glaucoma Stereo Analysis Study. PLoS One 9:e99138

9. Savini G, Zanini M, Carelli V, Sadun AA, Ross-Cisneros FN, Barboni P (2005) Correlation between retinal nerve fibre layer thickness and optic nerve head size: an optical coherence tomography study. Br J Ophthalmol 89:489-492
10. Budenz DL, Anderson DR, Varma R, Schuman J, Cantor L, Savell J, Greenfield DS, Patella VM, Quigley HA, Tielsch J (2007) Determinants of normal retinal nerve fiber layer thickness measured by Stratus OCT. Ophthalmology 114:1046-1052

11. Mardin CY, Horn FK (1998) Influence of optic disc size on the sensitivity of the Heidelberg Retina Tomograph. Graefes Arch Clin Exp Ophthalmol 236:641-645

12. Huang D, Chopra V, Lu AT, Tan O, Francis B, Varma R, Advanced Imaging for Glaucoma Study-AIGS Group (2012) Does optic nerve head size variation affect circumpapillary retinal nerve fiber layer thickness measurement by optical coherence tomography? Invest Ophthalmol Vis Sci 53:4990-4997

13. Danesh-Meyer HV, Ku JY, Papchenko TL, Jayasundera T, Hsiang JC, Gamble GD (2006) Regional correlation of structure and function in glaucoma, using the Disc Damage Likelihood Scale, Heidelberg Retina Tomograph, and visual fields. Ophthalmology 113:603-611

14. Danesh-Meyer HV, Gaskin BJ, Jayusundera T, Donaldson M, Gamble GD (2006) Comparison of disc damage likelihood scale, cup to disc ratio, and Heidelberg retina tomograph in the diagnosis of glaucoma. Br J Ophthalmol 90:437-441

15. Han JW, Cho SY, Kang KD (2014) Correlation between optic nerve parameters obtained using 3D nonmydriatic retinal camera and optical coherence tomography: interobserver agreement on the Disc damage likelihood scale. J Ophthalmol 2014:931738

16. Leske MC, Heijl A, Hussein M, Bengtsson B, Hyman L, Komaroff E, Early Manifest Glaucoma Trial Group (2003) Factors for glaucoma progression and the effect of treatment: the early manifest glaucoma trial. Arch Ophthalmol 121:48-56

17. Hornova J, Kuntz Navarro JB, Prasad A, Freitas DG, Nunes CM (2008) Correlation of disc damage likelihood scale, visual field, and Heidelberg Retina Tomograph II in patients with glaucoma. Eur J Ophthalmol 18:739-747

18. Abdul Majid AS, Kwag JH, Jung SH, Yim HB, Kim YD, Kang KD (2010) Correlation between disc damage likelihood scale and optical coherence tomography in the diagnosis of glaucoma. Ophthalmologica 224:274-282

19. Suzuki Y, Iwase A, Araie M, Yamamoto T, Abe H, Shirato S, Kuwayama Y, Mishima HK, Shimizu H, Tomita G, Inoue Y, Kitazawa Y, Tajimi Study Group (2006) Risk factors for openangle glaucoma in a Japanese population: the Tajimi Study. Ophthalmology 113:1613-1617

20. Perera SA, Wong TY, Tay WT, Foster PJ, Saw SM, Aung T (2010) Refractive error, axial dimensions, and primary open-angle glaucoma: the Singapore Malay Eye Study. Arch Ophthalmol 128:900905

21. Kuzin AA, Varma R, Reddy HS, Torres M, Azen SP, Los Angeles Latino Eye Study Group (2010) Ocular biometry and open-angle glaucoma: the Los Angeles Latino Eye Study. Ophthalmology 117: 1713-1719

22. Marcus MW, de Vries MM, Junoy Montolio FG, Jansonius NM (2011) Myopia as a risk factor for open-angle glaucoma: a systematic review and meta-analysis. Ophthalmology 118:1989-94.e2 